\title{
Correction: P53-regulated miR-320a targets PDL1 and is downregulated in malignant mesothelioma
}

Caterina Costa (1), Paola Indovina, Eliseo Mattioli, Iris Maria Forte, Carmelina Antonella lannuzzi, Luca Luzzi, Cristiana Bellan, Simona De Summa (D), Enrico Bucci $\mathbb{D}$, Domenico Di Marzo, Marisa De Feo, Luciano Mutti $\mathbb{B}$, Francesca Pentimalli(i) and Antonio Giordano (1)

Correction to: Cell Death \& Disease https://doi.org/10.1038/s41419-020-02940-w published online 14 September 2020

Owing to an error in production, the original version of this Article contained an error in the author affiliations.

Author Francesca Pentimalli was incorrectly associated with Histopathological Unit, IRCCS-Istituto Tumori "Giovanni Paolo II", Viale Orazio Flacco 65, 70124 Bari,
Italy. The author's actual affiliation is Cell Biology and Biotherapy Unit, Istituto Nazionale Tumori-IRCCSFondazione G. Pascale, I-80131 Napoli, Italy.

We apologise for this error, and confirm that it this has now been corrected in both the PDF and HTML versions of the Article.

Published online: 16 October 2020 